\title{
Bulk-boundary asymptotic equivalence of two strict deformation quantizations
}

\author{
Valter Moretti $^{1}$ (D) Christiaan J. F. van de Ven ${ }^{1,2}$
}

Received: 9 May 2020 / Revised: 14 September 2020 / Accepted: 21 September 2020 /

Published online: 1 October 2020

(c) The Author(s) 2020

\begin{abstract}
The existence of a strict deformation quantization of $X_{k}=S\left(M_{k}(\mathbb{C})\right)$, the state space of the $k \times k$ matrices $M_{k}(\mathbb{C})$ which is canonically a compact Poisson manifold (with stratified boundary), has recently been proved by both authors and Landsman (Rev Math Phys 32:2050031, 2020. https://doi.org/10.1142/S0129055X20500312). In fact, since increasing tensor powers of the $k \times k$ matrices $M_{k}(\mathbb{C})$ are known to give rise to a continuous bundle of $C^{*}$-algebras over $I=\{0\} \cup 1 / \mathbb{N} \subset[0,1]$ with fibers $A_{1 / N}=$ $M_{k}(\mathbb{C})^{\otimes N}$ and $A_{0}=C\left(X_{k}\right)$, we were able to define a strict deformation quantization of $X_{k}$ à la Rieffel, specified by quantization maps $Q_{1 / N}: / \tilde{A}_{0} \rightarrow A_{1 / N}$, with $\tilde{A}_{0}$ a dense Poisson subalgebra of $A_{0}$. A similar result is known for the symplectic manifold $S^{2} \subset \mathbb{R}^{3}$, for which in this case the fibers $A_{1 / N}^{\prime}=M_{N+1}(\mathbb{C}) \cong B\left(\operatorname{Sym}^{N}\left(\mathbb{C}^{2}\right)\right)$ and $A_{0}^{\prime}=C\left(S^{2}\right)$ form a continuous bundle of $C^{*}$-algebras over the same base space $I$, and where quantization is specified by (a priori different) quantization maps $Q_{1 / N}^{\prime}: \tilde{A}_{0}^{\prime} \rightarrow$ $A_{1 / N}^{\prime}$. In this paper, we focus on the particular case $X_{2} \cong B^{3}$ (i.e., the unit three-ball in $\left.\mathbb{R}^{3}\right)$ and show that for any function $f \in \tilde{A}_{0}$ one has $\left.\lim _{N \rightarrow \infty}||\left(Q_{1 / N}(f)\right)\right|_{\operatorname{Sym}^{N}\left(\mathbb{C}^{2}\right)}-$ $Q_{1 / N}^{\prime}\left(\left.f\right|_{S^{2}}\right) \|_{N}=0$, where $\operatorname{Sym}^{N}\left(\mathbb{C}^{2}\right)$ denotes the symmetric subspace of $\left(\mathbb{C}^{2}\right)^{N \otimes}$. Finally, we give an application regarding the (quantum) Curie-Weiss model.
\end{abstract}

\section{Contents}

1 Introduction . . . . . . . . . . . . . . . . . . . . . . . . . . . . . . 2942

1.1 Strict deformation quantization maps . . . . . . . . . . . . . . . . . . . . . . . . . . . . 2942

1.2 Spin systems and generalizations . . . . . . . . . . . . . . . . . . . . . . . . . . . . . 2944

$\bowtie$ Valter Moretti

valter.moretti@unitn.it

Christiaan J. F. van de Ven

christiaan.vandeven@unitn.it

1 Department of Mathematics, University of Trento, INFN-TIFPA, via Sommarive 14, 38123

Povo, Trento, Italy

2 Istituto Nazionale di Alta Matematica, Rome, Italy 
2 Interplay of bulk quantization map $Q_{1 / N}$ and boundary quantization map $Q_{1 / N}^{\prime} \ldots \ldots$. . . 2948

2.1 Preparatory results on $Q_{1 / N}^{\prime}$ and harmonic polynomials . . . . . . . . . . . . . . . . 2948

2.2 The main theorem . . . . . . . . . . . . . . . . . . . . . . . . . 2950

2.3 Subsidiary technical results . . . . . . . . . . . . . . . . . . . . . . . 2955

3 Application to the quantum Curie-Weiss model . . . . . . . . . . . . . . . . . . . . . . . . . 2958

Appendix A: Continuous bundle of $C^{*}$-algebras . . . . . . . . . . . . . . . . . . . . . . . . . . 2960

Appendix B: Coherent spin states . . . . . . . . . . . . . . . . . . . . . . . . . . . . . . . . . 2961

References . . . . . . . . . . . . . . . . . . . . . . . . . . . 2962

\section{Introduction}

An important field of research within mathematical physics concerns the relation between classical theories viewed as limits of quantum theories, for example, classical mechanics of a particle on the phase space $\mathbb{R}^{2 n}$ versus quantum mechanics on the Hilbert space $L^{2}\left(\mathbb{R}^{n}\right)$, or classical thermodynamics of a spin system versus statistical mechanics of a quantum spin system on a finite lattice [14]. In these examples, the relation between both (different) theories can be described by a continuous bundle of algebras of observables equipped with certain quantization maps. A modern way establishing a link between both theories is based on the concept of strict deformation quantization, i.e., the mathematical formalism that describes the transition from a classical theory to a quantum theory [13,22,23] in terms of deformations of (commutative) Poisson algebras (representing the classical theory) into non-commutative $C^{*}$ algebras characterizing the quantum theory.

\subsection{Strict deformation quantization maps}

Let us focus to the first known example starting from the familiar classical phase space $\mathbb{R}^{2 n}$. For convenience, we only consider the Poisson algebra of smooth compactly supported functions $f \in C_{c}^{\infty}\left(\mathbb{R}^{2 n}\right)$ where the Poisson structure is the one associated with the natural symplectic form $\sum_{j=1}^{n} d p_{j} \wedge d q^{j}$ [17]. In order to relate $C_{c}^{\infty}\left(\mathbb{R}^{2 n}\right)$ to a quantum theory described on some Hilbert space, one needs to deform $C_{c}^{\infty}\left(\mathbb{R}^{2 n}\right)$ into non-commutative $C^{*}$-algebras exploiting a family of quantization maps. Berezin proposed the quantization maps [3]

$$
\begin{aligned}
Q_{\hbar}: C_{c}^{\infty}\left(\mathbb{R}^{2 n}\right) & \rightarrow B_{0}\left(L^{2}\left(\mathbb{R}^{n}\right)\right) \\
Q_{\hbar}(f) & =\int_{\mathbb{R}^{2 n}} \frac{d^{n} p d^{n} q}{(2 \pi \hbar)^{n}} f(p, q)\left|\phi_{\hbar}^{(p, q)}\right\rangle\left\langle\phi_{\hbar}^{(p, q)}\right|,
\end{aligned}
$$

where $\hbar \in(0,1] ; B_{0}(H)$ is the $C^{*}$-algebra of compact operators on the Hilbert space $H=L^{2}\left(\mathbb{R}^{n}\right)$, and for each point $(p, q) \in \mathbb{R}^{2 n}$, the (projection) operator $\left|\phi_{\hbar}^{(p, q)}\right\rangle\left\langle\phi_{\hbar}^{(p, q)}\right|: L^{2}\left(\mathbb{R}^{n}\right) \rightarrow L^{2}\left(\mathbb{R}^{n}\right)$ is induced by the normalized wave functions, where $x \in \mathbb{R}^{n}$,

$$
\phi_{\hbar}^{(p, q)}(x)=(\pi \hbar)^{-n / 4} e^{-i p q / 2 \hbar} e^{-i p x / \hbar} e^{-(x-q)^{2} / 2 \hbar}, \quad \phi_{\hbar}^{(p, q)} \in L^{2}\left(\mathbb{R}^{n}\right),
$$


defining the well-known (Schrodinger) coherent states. Inspired by Dixmier's concept of a continuous bundle [7], Rieffel showed that [22,23]

1. The fibers $A_{0}=C_{0}\left(\mathbb{R}^{2 n}\right)$ and $A_{\hbar}=B_{0}\left(L^{2}\left(\mathbb{R}^{n}\right)\right), h \in(0,1]$, can be combined into a (locally non-trivial) continuous bundle $A$ of $C^{*}$-algebras over $I=[0,1]$;

2. $\tilde{A}_{0}=C_{c}^{\infty}\left(\mathbb{R}^{2 n}\right)$ is a dense Poisson subalgebra of $A_{0}$.

3. Each quantization map $Q_{\hbar}: \tilde{A}_{0} \rightarrow A_{\hbar}$ is linear, and if we also define $Q_{0}: \tilde{A}_{0} \hookrightarrow$ $A_{0}$ as the inclusion map, then the ensuing family $Q=\left(Q_{\hbar}\right)_{\hbar \in I}$ satisfies:

(a) Each map $Q_{\hbar}$ is self-adjoint, i.e., $Q_{\hbar}(\bar{f})=Q_{\hbar}(f)^{*}$ (where $f^{*}(x)=\overline{f(x)}$ ).

(b) For each $f \in \tilde{A}_{0}$, the following cross section of the bundle is continuous:

$$
\begin{aligned}
& 0 \rightarrow f ; \\
& \left.\hbar \rightarrow Q_{\hbar}(f) \quad(\hbar \in I \backslash\{0\})\right) .
\end{aligned}
$$

(c) Each pair $f, g \in \tilde{A}_{0}$ satisfies the Dirac-Groenewold-Rieffel condition:

$$
\lim _{\hbar \rightarrow 0}\left\|\frac{i}{\hbar}\left[Q_{\hbar}(f), Q_{\hbar}(g)\right]-Q_{\hbar}(\{f, g\})\right\|_{\hbar}=0 .
$$

This led to the general concept of a strict deformation of a Poisson manifold $X[13,22]$, which we here state in the case of interest to us in which $X$ is compact, or more generally in which $X$ is a manifold with stratified boundary [15,19]. In that case, the space $I$ in which $\hbar$ takes values cannot be all of $[0,1]$, but should be a subspace $I \subset[0,1]$ thereof that at least contains 0 as an accumulation point. This is assumed in what follows. Furthermore, the Poisson bracket on $X$ is denoted, as usual, by $\{\cdot, \cdot\}: C^{\infty}(X) \times C^{\infty}(X) \rightarrow C^{\infty}(X)$ (where the smooth space $C^{\infty}(X)$ is suitably defined when $X$ is a more complicated object than a compact smooth manifold as we shall say shortly).

Definition 1.1 A strict deformation quantization (according to [14] ${ }^{1}$ ) of a compact Poisson manifold $X$ consists of an index space $I \subset[0,1]$, including 0 as accumulation point, for $\hbar$ as detailed above, as well as:

- A continuous bundle of unital $C^{*}$-algebras $\left(A_{\hbar}\right)_{\hbar \in I}$ over $I$ with $A_{0}=C(X)$ equipped with the standard commutative $C^{*}$-algebra structure with respect to the norm $\|\cdot\|_{\infty}$;

- A $\|\cdot\|_{\infty}$-dense Poisson suabalgebra $\tilde{A}_{0} \subseteq C^{\infty}(X) \subset A_{0}$ (on which $\{\cdot, \cdot\}$ is defined);

- A family $Q=\left(Q_{\hbar}\right)_{\hbar \in I}$ of linear maps $Q_{\hbar}: \tilde{A}_{0} \rightarrow A_{\hbar}$ indexed by $\hbar \in I$ (called quantization maps) such that $Q_{0}$ is the inclusion map $\tilde{A}_{0} \hookrightarrow A_{0}$, and the above conditions (a)-(c) hold, as well as $Q_{\hbar}\left(1_{X}\right)=1_{A_{\hbar}}$ (the unit of $A_{\hbar}$ ).

\footnotetext{
1 We stress that the some authors adopt a different notion of strict (deformation) quantization. For example, in Rieffel's approach the same (quantum) algebra is used and the product, depending on $\hbar$, is deformed $[22,23]$. In this setting, the image of the quantization map lies in an algebra with a "new" product. We instead follow the definitions introduced by Landsman [13,14] (who adapted Rieffel's original ideas), where for $\hbar>0$ he uses the non-commutative algebras with their intrinsic product independent of $\hbar$. The $\hbar$ dependence in turn is put in the quantization map itself. The term "strict deformation quantization" we use in this paper is therefore related to Landsman's notion of quantization.
} 
It follows from the definition of a continuous bundle of $C^{*}$-algebras that two continuity properties hold

$$
\lim _{\hbar \rightarrow 0}\left\|Q_{\hbar}(f)\right\|_{\hbar}=\|f\|_{\infty}
$$

and

$$
\lim _{\hbar \rightarrow 0}\left\|Q_{\hbar}(f) Q_{\hbar}(g)-Q_{\hbar}(f g)\right\|_{\hbar}=0
$$

hold automatically $[7,13,14]$.

\subsection{Spin systems and generalizations}

Mean-field quantum spin systems ${ }^{2}$ fit into this framework. There, the index set $I$ is given by $(0 \notin \mathbb{N}:=\{1,2,3, \ldots\})$

$$
I=\{1 / N \mid N \in \mathbb{N}\} \cup\{0\} \equiv(1 / \mathbb{N}) \cup\{0\},
$$

with the topology inherited from $[0,1]$. That is, we put $\hbar=1 / N$, where $N \in \mathbb{N}$ is interpreted as the number of sites of the model; our interest is the limit $N \rightarrow \infty$. In the framework of $C^{*}$-algebraic quantization theory, the analogy between the "classical" limit $\hbar \rightarrow 0$ in typical examples from mechanics (see, e.g., our first example [10]) and the "thermodynamic" limit $N \rightarrow \infty$ in typical quantum spin systems (see, e.g., $[15,16])$ is developed in detail in [14]. We remark that the limit $N \rightarrow \infty$ can be taken in two entirely different ways, which depends on the class of observables one considers, namely either quasi-local observables or macroscopic observables. The former are the ones traditionally studied for quantum spin systems, but the latter relate these systems to strict deformation quantization, since macroscopic observables are precisely defined by (quasi-)symmetric sequences which form the continuous cross sections of a continuous bundle of $C^{*}$-algebras. This continuous bundle of $C^{*}$-algebras is defined over base space $I$ given by (1.9) with fibers

$$
\begin{aligned}
A_{0} & =C\left(S\left(M_{k}(\mathbb{C})\right) \equiv C\left(X_{k}\right) ;\right. \\
A_{1 / N} & =M_{k}(\mathbb{C})^{\otimes N} \cong M_{k^{N}}(\mathbb{C}),
\end{aligned}
$$

and continuity structure specified by continuous cross sections which are thus given by all quasi-symmetric sequences [15] [14, Ch.10]. ${ }^{3}$ We refer to the appendix for some useful definitions or to [15] for a more comprehensive explanation. The space $X_{k}=S\left(M_{k}(\mathbb{C})\right) \subset \mathbb{R}^{k^{2}-1}$ has the structure of a compact Poisson manifold with

\footnotetext{
2 A typical example of a mean-field quantum spin system is the Curie-Weiss model (see, for example, $[1,6,11,25-27]$ and references therein).

3 The same result holds for an arbitary unital $C^{*}$-algebra $B$ playing the role of the matrix algebra $M_{k}(\mathbb{C})$ in the above setting [14]. 
stratified boundary. The space $C^{\infty}\left(X_{k}\right)$ is here made of the restrictions to $X_{k}$ of the smooth functions in $\mathbb{R}^{k^{2}-1}$, and the Poisson bracket is the restriction $\mathbf{x} \in X_{k}$

$$
\{f, g\}(\mathbf{x})=\sum_{a, b, c=1}^{k^{2}-1} C_{a b}^{c} x_{c} \frac{\partial f}{\partial x_{a}} \frac{\partial g}{\partial x_{b}}, \quad \mathbf{x} \in \mathbb{R}^{k^{2}-1}
$$

for $f, g \in C^{\infty}\left(\mathbb{R}^{k^{2}-1}\right)$ and where $C_{a b}^{c}$ are the structure constants of $S U(k)$ (see Sect. 2.3 of [15] for details). In turn, the Poisson algebra $\tilde{A}_{0}$ dense in $A_{0}=C\left(X_{k}\right)$ is made of the restrictions to $X_{k}$ of the polynomials in the $k^{2}-1$ coordinates of $\mathbb{R}^{k^{2}-1}$

Let us pass to describe $Q_{1 / N}$. Each polynomial $p$ of degree $L$ uniquely corresponds to a polynomial of symmetric elementary tensors of the form $b_{j_{1}} \otimes_{S} \cdots \otimes_{S} b_{j_{L}}$, where $i b_{1}, \ldots, i b_{k^{2}-1}$ form a basis of the Lie algebra of $S U(k)$. That is the image of $p$ according to $Q_{1 / N}$. More precisely, if

$$
p_{L}\left(x_{1}, \ldots, x_{k^{2}-1}\right)=x_{j_{1}} \cdots x_{j_{L}} \quad \text { where } j_{1}, \ldots, j_{L} \in\left\{1,2, \ldots, k^{2}-1\right\}
$$

the quantization maps $Q_{1 / N}: \tilde{A}_{0} \rightarrow M_{k}(\mathbb{C})^{N}$ act as (see the appendix for $S_{L, N}$ )

$$
\begin{aligned}
Q_{1 / N}\left(p_{L}\right) & = \begin{cases}S_{L, N}\left(b_{j_{1}} \otimes_{s} \cdots \otimes_{s} b_{j_{L}}\right), & \text { if } N \geq L \\
0, & \text { if } N<L,\end{cases} \\
Q_{1 / N}(1) & =\underbrace{I_{k} \otimes \cdots \otimes I_{k}}_{N \text { times }} \cdot,
\end{aligned}
$$

and more generally, they are defined as the unique continuous and linear extensions of the written maps.

It has been shown in [15] that the quantization maps $Q_{1 / N}$ satisfy all the axioms of Definition 1.1. ${ }^{4}$ These data together imply the existence of a strict deformation quantization of the Poisson manifold $X_{k}=S\left(M_{k}(\mathbb{C})\right.$ ) (see [15, Theorem 3.4] for a detailed proof).

We specialize these models to the case $k=2$. One-dimensional quantum spin systems arising in that way are widely studied in (condensed matter) physics, but also in mathematical physics they form an important field of research, especially in view of spontaneous symmetry breaking (SSB). One tries to calculate quantities like the free energy, or the entropy of the system in question and considers their thermodynamic limit as the number of sites $N$ increase to infinity [16]. For this reason, the case $k=2$ is already of huge interest, since each site of such a spin chain is exactly described by the algebra of $(2 \times 2)$-matrices. On the other hand, the Bloch sphere $S^{2}$ acting as a classical phase space which describes a physical system may be a spin system of total spin $j$, but it can also be a collection of $n$ two-level atoms [2] corresponding to a spin chain of $n$ sites, which is, for example, the case for the quantum Curie-Weiss model

\footnotetext{
4 In particular, the quantization maps define (quasi-)symmetric sequences and hence macroscopic observables.
} 
[15]. Inspired by that model, which admits a classical limit ${ }^{5}$ on $S^{2}$ (i.e., the smooth boundary of $X_{2}=S\left(M_{2}(\mathbb{C})\right) \cong B^{3}$, where $B^{3}$ denotes the closed unit ball in $\mathbb{R}^{3}$ ), we asked ourselves if the quantization maps $Q_{1 / N}$ quantizing $X_{2}$ could in general be related to another well-known strict deformation quantization of $S^{2}$ whose details are explained in what follows. ${ }^{6}$

From the mathematical side, we observe that $k=2$ is the unique case where $X_{k}$ admits a smooth boundary, as said $X_{2}=B^{3}$ and $\partial X_{2}=S^{2}$. Furthermore, $S^{2}$ is a Poisson submanifold of $B^{3}$, when the latter is equipped with the Poisson structure (1.16) specialized to $k=2$, so that $C_{b c}^{a}=\epsilon_{a b c}$. This is because $S^{2}$ (and also $B^{3}$ ) is invariant under the flow of the Hamilton vector fields of $\mathbb{R}^{k^{2}-1}$ constructed out of the Poisson bracket (1.16). For $k=2$, we precisely have

$$
\left.\{f, g\}^{\left(B^{3}\right)}\right|_{S^{2}}=\left\{\left.f\right|_{S^{2}},\left.g\right|_{S^{2}}\right\}^{\left(S^{2}\right)} \quad \text { if } f, g \in \tilde{A}_{0},
$$

with obvious notation. In particular,

$$
\{f, g\}^{\left(B^{3}\right)}(\mathbf{x})=\sum_{a, b, c=1}^{3} \epsilon_{a b c} x_{c} \frac{\partial f}{\partial x_{a}} \frac{\partial g}{\partial x_{b}}, \quad \mathbf{x} \in B^{3} .
$$

This paper only concerns the case $k=2$. In a sense, we promote at quantum level the illustrated interplay of the two symplectic structures of $X_{2}=B^{3}$ and $\partial B^{3}=S^{2}$. As a matter of fact, we consider the quantization elements $Q_{1 / N}(f) \in B\left(\left(\mathbb{C}^{2}\right)^{N \otimes}\right)$ under the maps (1.13)-(1.14) referred to the symplectic structure of $B^{3}$. Next we restrict the operators $Q_{1 / N}(f)$ to a suitable common invariant subspace of $\left(\mathbb{C}^{2}\right)^{N \otimes}$. It turns out that, for large $N$, these restricted operators correspond to the image of another quantization map $Q_{1 / N}^{\prime}$ acting on $C\left(\partial B^{3}\right)$ and referring to the symplectic structure of $\partial B^{3}$.

The said invariant subspace ${ }^{7}$ is $\operatorname{Sym}^{N}\left(\mathbb{C}^{2}\right) \subset\left(\mathbb{C}^{2}\right)^{N \otimes}$, for which the corresponding algebras $B\left(\operatorname{Sym}^{N}\left(\mathbb{C}^{2}\right)\right.$ ) exactly correspond to the fibers (for $N \neq 0$ ) of another continuous bundle of $C^{*}$-algebras given by (1.17)-(1.18). It is a well-known fact that these fibers together with quantization maps (1.21)-(1.22) below give rise to a strict deformation quantization of $S^{2}[5,13,18]$ according to Definition 1.1.

Indicating the algebra of bounded operators by $B\left(\operatorname{Sym}^{N}\left(\mathbb{C}^{2}\right)\right)$, it is known $[14$, Theorem 8.1] that

$$
\begin{aligned}
A_{0}^{\prime} & =C\left(S^{2}\right) \\
A_{1 / N}^{\prime} & =M_{N+1}(\mathbb{C}) \cong B\left(\operatorname{Sym}^{N}\left(\mathbb{C}^{2}\right)\right),
\end{aligned}
$$

\footnotetext{
$\left.\overline{5 \text { This means that }\left\langle\Psi_{N}^{(0)}, Q_{1 / N}(f)\right.} \Psi_{N}^{(0)}\right\rangle$ admits a limit as $N \rightarrow \infty$ for any function $f \in \tilde{A}_{0}$, and $\Psi_{N}^{(0)}$ the ground state eigenvector of the quantum CW Hamiltonian (see [15, Theorem 4.1] for details).

${ }^{6}$ Of course, one can always try to restrict $\tilde{A}_{0}$ to $\tilde{A}_{0}^{\prime}$, but in that case the same manifolds are quantized which is not of particular new interest.

7 This space is clearly invariant under the maps (1.13)-(1.14).
} 
are the fibers of a continuous bundle of $C^{*}$-algebras over the same base space $I$ as in (1.9) whose continuous cross sections are given by all sequences $\left(a_{1 / N}\right)_{N \in \mathbb{N}} \in$ $\Pi_{n \in \mathbb{N}} A_{1 / N}^{\prime}$ for which $a_{0} \in C\left(S^{2}\right)$ and $a_{1 / N} \in M_{N+1}(\mathbb{C})$ and such that the sequence $\left(a_{1 / N}\right)_{N \in \mathbb{N}}$ is asymptotically equivalent to $\left(Q_{1 / N}^{\prime}\left(a_{0}\right)\right)_{N \in \mathbb{N}}$, in the sense that

$$
\lim _{N \rightarrow \infty}\left\|a_{1 / N}-Q_{1 / N}^{\prime}\left(a_{0}\right)\right\|_{N}=0 .
$$

Here, the symbol $Q_{1 / N}^{\prime}$ denotes the quantization maps

$$
Q_{1 / N}^{\prime}: \tilde{A}_{0}^{\prime} \rightarrow A_{1 / N}^{\prime}
$$

where $\tilde{A}_{0}^{\prime} \subset C^{\infty}\left(S^{2}\right) \subset A_{0}^{\prime}$ is the dense Poisson subalgebra made of polynomials in three real variables restricted the sphere $S^{2}$. The maps $Q_{1 / N}^{\prime}$ are defined by ${ }^{8}$ the integral computed in weak sense

$$
Q_{1 / N}^{\prime}(p):=\frac{N+1}{4 \pi} \int_{S^{2}} p(\Omega)|\Omega\rangle\left\langle\left.\Omega\right|_{N} d \Omega,\right.
$$

where $p$ denotes an arbitrary polynomial restricted to $S^{2}, \mathrm{~d} \Omega$ indicates the unique $S O(3)$-invariant Haar measure on $S^{2}$ with $\int_{S^{2}} \mathrm{~d} \Omega=4 \pi$, and $|\Omega\rangle\left\langle\left.\Omega\right|_{N} \in\right.$ $B\left(\operatorname{Sym}^{N}\left(\mathbb{C}^{2}\right)\right)$ are so-called $N$ coherent spin states defined in "Appendix B." In particular, if 1 is the constant function $1(\Omega)=1,\left(\Omega \in S^{2}\right)$, and $1_{N}$ is the identity on $A_{1 / N}^{\prime}=B\left(\operatorname{Sym}^{N}\left(\mathbb{C}^{2}\right)\right)$, the previous definition implies

$$
Q_{1 / N}^{\prime}(1)=1_{N}
$$

Indeed, it can be shown that the quantization maps (1.21)-(1.22) satisfy the axioms of Definition 1.1, which implies the existence of a strict deformation quantization of $S^{2} .9$ These quantization maps, constructed from a family coherent states (as opposed to the maps (1.13)-(1.14) which are defined in a complete different way), also define a socalled Berezin quantization [13] for which (B.3) typically holds as well as positivity, in that $Q_{1 / N}(f) \geq 0$ if $f \geq 0$ almost everywhere on $S^{2}$.

The main result of this work is an asymptotic relation connecting the bulk and the boundary quantization maps:

$$
\left\|\left|Q_{1 / N}(p)\right|_{S y m(N)}\left(\left(\mathbb{C}^{2}\right)^{N \otimes}\right)-Q_{1 / N}^{\prime}\left(\left.p\right|_{S^{2}}\right)\right\|_{N} \rightarrow 0 \text { for } N \rightarrow+\infty, \quad p \in \tilde{A}_{0}
$$

established in Theorem 2.3. We stress that the validity of the Dirac-GroenewoldRieffel condition (1.6) for both maps is possible just thank to (1.15).

\footnotetext{
8 Equivalent definitions of these quantization maps are used in the literature, see, e.g., [14,18].

9 We remark that $S^{2}$ is a special case of a regular integral coadjoint orbit in the dual of the Lie algebra associated with $S U(2)$, which can be identified with $\mathbb{R}^{3}$. In fact, this theory can be generalized to arbitrary compact connected Lie groups [13].
} 
The plan of this paper is as follows. In section 2, we state and prove our main theorem (Theorem 2.3) establishing a connection between the strict deformation quantization of $X_{2}$ and the one of $S^{2}$ defined above. We show that the quantization maps $Q_{1 / N}$ defined by (1.13)-(1.14) whose images are restricted to $\operatorname{Sym}^{N}\left(\mathbb{C}^{2}\right)$ satisfy the identity above with respect to the other quantization map $Q_{1 / N}^{\prime}$. In section 3 , we apply our theorem to the Curie-Weiss model which links the corresponding quantum Hamiltonian to its classical counterpart on the sphere. In the appendix, we provide a comprehensive overview of useful definitions.

\section{Interplay of bulk quantization map $Q_{1 / N}$ and boundary quantization map $Q_{1 / N}^{\prime}$}

In order to arrive at the main theorem of this paper, we first introduce some vector spaces. We let $P_{N}$ to be the complex vector space of polynomials in the variables $x, y, z \in \mathbb{R}^{3}$ of degree $\leq N$ where $N \geq 1$, and let $P_{N}\left(S^{2}\right)$ be the vector space made of the restrictions to $S^{2}$ of those polynomials.

\subsection{Preparatory results on $Q_{1 / N}^{\prime}$ and harmonic polynomials}

Definition (1.21) can actually be stated replacing the polynomial $p$ by a generic $f \in C\left(S^{2}\right)$, though its meaning as a quantization map is valid for the domain of the polynomials restricted to $S^{2}$ as indicated in (1.21). The map associating $f \in C\left(S^{2}\right)$ with

$$
\begin{aligned}
& Q_{1 / N}^{\prime}(f): \operatorname{Sym}^{N}\left(\mathbb{C}^{2}\right) \rightarrow \operatorname{Sym}^{N}\left(\mathbb{C}^{2}\right) \\
& Q_{1 / N}^{\prime}(f):=\frac{N+1}{4 \pi} \int_{S^{2}} f(\Omega)|\Omega\rangle\left\langle\left.\Omega\right|_{N} \mathrm{~d} \Omega,\right.
\end{aligned}
$$

is well defined and it is surjective on $B\left(\operatorname{Sym}^{N}\left(\mathbb{C}^{2}\right)\right)$ since, for every $A: \operatorname{Sym}^{N}\left(\mathbb{C}^{2}\right) \rightarrow$ $\operatorname{Sym}^{N}\left(\mathbb{C}^{2}\right)$, there exists a function $p \in P_{N}\left(S^{2}\right)$ such that $A=Q_{1 / N}^{\prime}(p)$. Indeed, that the function

$$
p(\Omega):=\operatorname{tr}\left(A \Delta_{N}^{(1)}(\Omega)\right)
$$

where $\Omega \in S^{2}$ and $\Omega \mapsto \Delta_{N}^{(1)} \in \operatorname{Sym}^{N}\left(\mathbb{C}^{2}\right)$ is defined by Definition (2.6) in [12], defines a polynomial on the sphere, i.e.,

$$
\operatorname{tr}\left(A \Delta_{N}^{(1)}\right) \in P_{N}\left(S^{2}\right)
$$

In particular, we realize that the linear map (2.2) cannot be injective on the domain $C\left(S^{2}\right)$ since this space is infinite-dimensional, whereas the co-domain is finitedimensional. Nevertheless, if restricting the domain to $P_{N}\left(S^{2}\right)$, the said map turns out to be bijective. 


\section{Proposition 2.1 The map}

$$
P_{N}\left(S^{2}\right) \ni p \mapsto Q_{1 / N}^{\prime}(p):=\frac{N+1}{4 \pi} \int_{S^{2}} p(\Omega)|\Omega\rangle\left\langle\left.\Omega\right|_{N} d \Omega \in B\left(\operatorname{Sym}^{N}\left(\mathbb{C}^{2}\right)\right)\right.
$$

is a bijection for $N>1$.

Proof The said map is obviously surjective, as already observed, because, by defining $p(\Omega):=\operatorname{tr}\left(A \Delta_{N}^{(1)}(\Omega)\right)$ for $A \in B\left(\operatorname{Sym}^{(N)}\left(\mathbb{C}^{2}\right)\right)$, we have $A=Q_{1 / N}^{\prime}(p)$. Let us prove injectivity. It is well known [21] that $\operatorname{dim}\left(P_{N}\left(S^{2}\right)\right)=(N+1)^{2}$ if $N>1$. On the other hand, $\operatorname{dim}\left(B\left(\operatorname{Sym}^{(N)}\left(\mathbb{C}^{2}\right)\right)\right)=(N+1)^{2}$ as one immediately proves. As $\operatorname{dim}\left(B\left(\operatorname{Sym}^{(N)}\left(\mathbb{C}^{2}\right)\right)\right)=\operatorname{dim}\left(P_{N}\left(S^{2}\right)\right)<+\infty$, surjectivity implies injectivity from elementary results of linear algebra.

Going back to Weyl, let us recall a few results on the theory of $S O$ (3) representations of polynomials restricted to the unit sphere. The group $S O(3)$ admits a natural representation on $P_{N}\left(S^{2}\right)$ given by

$$
S O(3) \ni \mathbb{R} \mapsto \rho_{R}, \quad\left(\rho_{R} p\right)(\Omega):=p\left(R^{-1} \Omega\right) \quad \forall p \in P_{N}\left(S^{2}\right), \forall \Omega \in S^{2} .
$$

In turn, the space $P_{N}\left(S^{2}\right)$ admits a direct decomposition into invariant and irreducible subspaces under the action of $\rho$, viz.

$$
P_{N}\left(S^{2}\right)=\bigoplus_{j=0,1, \ldots, N} P_{N}^{(j)}\left(S^{2}\right)
$$

Each subspace $P_{N}^{(j)}\left(S^{2}\right)$ consists of the restrictions to $S^{2}$ of the homogeneous polynomials of order $j$ that are also harmonic functions. $P_{N}^{(j)}\left(S^{2}\right)$ has dimension $2 j+1$.

Example 2.2 If $N=2$,

$$
P_{2}\left(S^{2}\right)=P_{2}^{(0)}\left(S^{2}\right) \oplus P_{2}^{(1)}\left(S^{2}\right) \oplus P_{2}^{(2)}\left(S^{2}\right)
$$

In the right-hand side, the first subaspace is the span of the restriction to $S^{2}$ of the constant polynomial $p(x, y, z):=1$, the second one is the span of the restrictions of the three polynomials $p_{j}(x, y, z):=x_{j}, j=1,2,3$ where $x_{1}=x, x_{2}=y, x_{3}=z$, and the third one is the span of the restrictions to $S^{2}$ of five elements suitably chosen ${ }^{10}$ of the six polynomials $p_{i j}(x, y, z):=x_{i} x_{j}-\frac{1}{3} \delta_{i j}\left(x^{2}+y^{2}+z^{2}\right)$ for $i, j \in\{1,2,3\}$.

If $\rho_{R}^{(j)}$ is the restriction of $\rho_{R}$ to $P_{N}^{(j)}\left(S^{2}\right)$ and $\left\{p_{m}^{(j)}\right\}_{m=-j,-j+1, \ldots, j-1, j}$ is a basis of $P_{N}^{(j)}\left(S^{2}\right)$, we find

$$
\rho_{R}^{(j)} p_{m}^{(j)}=\sum_{m^{\prime}=-j}^{j} D_{m m^{\prime}}^{(j)}\left(R^{-1}\right) p_{m^{\prime}}^{(j)}
$$

10 The restrictions to $S^{2}$ of these six polynomials and the one of the above $p$ form a linearly dependent set. 
Each class of matrices $\left\{D^{(j)}(R)\right\}_{R \in S O(3)}$ defines an irreducible representation of $S O(3)$ in $\mathbb{C}^{2 j+1}$. These representations are completely fixed by their dimension, i.e., by $j$, up to equivalence given by similarity transformations, and different $j$ correspond to similarity inequivalent representations. Every irreducible representation of $S O(3)$ is unitarily equivalent to one of the $D^{(j)}$.

\subsection{The main theorem}

Before arriving at the main theorem of this paper, we recall that by construction the space $\tilde{A}_{0}$ is the complex vector space of polynomials in three variables on the closed unit ball $B^{3}$ which, in particular, contains all polynomials of $P_{M}(M \in \mathbb{N})$ restricted to $B^{3}$ [15]. In the proof of the theorem, we occasionally use the space $\tilde{A}_{0}$ as well as $P_{N}$, where the former is the domain of the quantization maps $Q_{1 / N}$, whereas the latter is used to underline the degree of the polynomial in question.

Theorem 2.3 If $p \in \tilde{A}_{0}$, then

$$
\left\|\left.Q_{1 / N}(p)\right|_{S y m} ^{(N)}\left(\left(\mathbb{C}^{2}\right)^{N \otimes}\right)-Q_{1 / N}^{\prime}\left(\left.p\right|_{S^{2}}\right)\right\|_{N} \rightarrow 0 \text { for } N \rightarrow+\infty,
$$

the (operator) norm being the one on $B\left(\operatorname{Sym}^{N}\left(\mathbb{C}^{2}\right)\right)$.

Remark 2.4 We stress that the result does not automatically imply that the cross sections (1.13)-(1.14) whose images are restricted to $\operatorname{Sym}^{N}\left(\mathbb{C}^{2}\right)$ are also continuous cross sections of the fibers defined in (1.17)-(1.18), since $f \in A_{0}=C\left(B^{3}\right)$ does not imply that $f \in A_{0}^{\prime}=C\left(S^{2}\right)$.

Proof We start the proof by discussing the interplay between the action of $S O$ (3) and the quantization maps $Q_{1 / N}$, defined in (1.13). We first focus on a homogeneous polynomial of order $M<N .{ }^{11}$ If $k_{1}, \ldots, k_{M}$ are taken in $\{1,2,3\}$ and

$$
p_{k_{1} \cdots k_{M}}(x, y, z):=x_{k_{1}} \cdots x_{k_{M}}
$$

the representation (2.6) implies that

$$
\left(\rho_{R} p_{k_{1} \cdots k_{M}}\right)(x, y, z)=\left(R_{U}^{-1}\right)_{k_{1}}^{j_{1}} \cdots\left(R_{U}^{-1}\right)_{k_{M}}^{j_{M}} p_{k_{1} \cdots k_{M}}(x, y, z) .
$$

We stress that when restricting to $S^{2}$, every $p_{m}^{(j)}$ is a linear combination of the restrictions of the polynomials $p_{k_{1} \cdots k_{M}}$ so that, by extending (2.9) by linearity and working on $p_{m}^{(j)},(2.9)$ must coincide with (2.7)

$$
\left(\rho_{R}^{(j)} p_{m}^{(j)}\right)(x, y, z)=\sum_{m^{\prime}=-j}^{j} D_{m m^{\prime}}^{(j)}\left(R^{-1}\right) p_{m^{\prime}}^{(j)}(x, y, z), \quad(x, y, z) \in S^{2}
$$

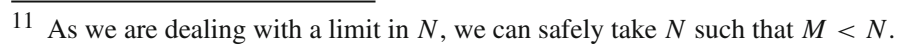


Since both sides are restrictions of homogeneous polynomials of the same degree $j$, this identity is valid also removing the constraint $(x, y, z) \in S^{2}$ :

$$
\left(\rho_{R}^{(j)} p_{m}^{(j)}\right)(x, y, z)=\sum_{m^{\prime}=-j}^{j} D_{m m^{\prime}}^{(j)}\left(R^{-1}\right) p_{m^{\prime}}^{(j)}(x, y, z), \quad(x, y, z) \in \mathbb{R}^{3},
$$

where now the $p_{m}^{(j)}$ are homogeneous polynomials in $P_{M}$ whose restrictions are the basis elements of $P_{M}^{(j)}\left(S^{2}\right)$ with the same name. We underline that for our quantization maps $Q_{1 / N}$ we need $p_{m}^{(j)}$ to be a polynomial in $\tilde{A}_{0}$, rather than in $P_{M}$. However, since $\tilde{A}_{0}$ contains all polynomials of $P_{M}$ restricted to $B^{3}$ which has non-empty interior, polynomials of $P_{M}$ are in one-to-one correspondence with those of $\tilde{A}_{0}$. Therefore, in view of (2.10) the same statement holds when we replace $(x, y, z) \in \mathbb{R}^{3}$ by $(x, y, z) \in$ $B^{3}$. Now, by definition of the quantization maps $Q_{1 / N}$ we know that

$$
Q_{1 / N}\left(p_{k_{1} \cdots k_{M}}\right)=S_{N, M}(\sigma_{k_{1}} \otimes \cdots \otimes \sigma_{k_{M}} \otimes \underbrace{I \otimes \cdots \otimes I}_{N-M \text { times }}) .
$$

Let us indicate by $R_{U} \in S O$ (3) the image of $U \in S U$ (2) through the universal covering homomorphism $\Pi: S U(2) \rightarrow S O(3)$. This covering homomorphism as is well known satisfies (using the summation convention on repeated indices)

$$
U \sigma_{j} U^{*}=\left(R_{U}^{-1}\right)_{j}{ }^{k} \sigma_{k}
$$

Remembering that $\operatorname{Sym}^{N}\left(\mathbb{C}^{2}\right)$ is invariant under the tensor representation $\underbrace{U \otimes \cdots \otimes U}_{N \text { times }}$, we have

$$
\begin{aligned}
& \left.\left.\left.\underbrace{U \otimes \cdots \otimes U}_{N \text { times }}\right|_{\operatorname{Sym}^{N}\left(\mathbb{C}^{2}\right)} Q_{1 / N}\left(p_{k_{1} \cdots k_{M}}\right)\right|_{\operatorname{Sym}^{N}\left(\mathbb{C}^{2}\right)} \underbrace{U^{*} \otimes \cdots \otimes U^{*}}_{N \text { times }}\right|_{\operatorname{Sym}^{N}\left(\mathbb{C}^{2}\right)} \\
& =\left.(\underbrace{U \otimes \cdots \otimes U}_{N \text { times }} Q_{1 / N}\left(p_{k_{1} \cdots k_{M}}\right) \underbrace{U^{*} \otimes \cdots \otimes U^{*}}_{N \text { times }})\right|_{\operatorname{Sym}^{N}\left(\mathbb{C}^{2}\right)} \\
& =\left.\left(R_{U}^{-1}\right)_{k_{1}}^{j_{1}} \cdots\left(R_{U}^{-1}\right)_{k_{M}}{ }^{j_{M}} Q_{1 / N}\left(p_{j_{1} \ldots j_{M}}\right)\right|_{\operatorname{Sym}^{N}\left(\mathbb{C}^{2}\right)} .
\end{aligned}
$$

Let us consider linear combinations $p_{m}^{(j)}$ of polynomials $p_{k_{1} \cdots k_{M}}$ whose restriction to $S^{2}$ define the basis element, indicated with the same symbol, $p_{m}^{(j)} \in P_{M}^{(j)}\left(S^{2}\right)$. Since the map $Q_{1 / N}$ is linear, from (2.10) we have 


$$
\begin{aligned}
& \left.(\underbrace{U \otimes \cdots \otimes U}_{N \text { times }} Q_{1 / N}\left(p_{m}^{(j)}\right) \underbrace{U^{*} \otimes \cdots \otimes U^{*}}_{N \text { times }})\right|_{\operatorname{Sym}^{N}\left(\mathbb{C}^{2}\right)} \\
& =\left.\sum_{m^{\prime}} D_{m m^{\prime}}^{(j)}\left(R^{-1}\right) Q_{1 / N}\left(p_{m^{\prime}}^{(j)}\right)\right|_{\operatorname{Sym}^{N}\left(\mathbb{C}^{2}\right)} .
\end{aligned}
$$

Let us now pass to the other quantization map $Q_{1 / N}^{\prime}$ observing that (2.12) and Proposition 2.1 entail

$$
\left.Q_{1 / N}\left(p_{m}^{(j)}\right)\right|_{\operatorname{Sym}^{N}\left(\mathbb{C}^{2}\right)}=Q_{1 / N}^{\prime}\left(q_{m}^{(j)}\right)=\frac{N+1}{4 \pi} \int_{S^{2}} q_{m}^{(j)}(\Omega)|\Omega\rangle\left\langle\left.\Omega\right|_{N} \mathrm{~d} \Omega\right.
$$

for some $q_{m}^{(j)} \in P_{N}\left(S^{2}\right)$ (where $N>M$ in general) is the unknown restriction to $S^{2}$ of a polynomial in $P_{N}$. Exploiting (2.12) and linearity, we find

$$
\begin{aligned}
& \left.\left.\left.\underbrace{U \otimes \cdots \otimes U}_{N \text { times }}\right|_{\operatorname{Sym}^{N}\left(\mathbb{C}^{2}\right)} Q_{1 / N}\left(p_{m}^{(j)}\right)\right|_{\mathrm{Sym}^{N}\left(\mathbb{C}^{2}\right)} \underbrace{U^{*} \otimes \cdots \otimes U^{*}}_{N \text { times }}\right|_{\operatorname{Sym}^{N}\left(\mathbb{C}^{2}\right)} \\
& =\frac{N+1}{4 \pi} \int_{S^{2}} \sum_{m^{\prime}} D_{m^{\prime}}^{(j)}\left(R^{-1}\right) q_{m^{\prime}}^{(j)}(\Omega)|\Omega\rangle\left\langle\left.\Omega\right|_{N} \mathrm{~d} \Omega .\right.
\end{aligned}
$$

Again, from (2.2) we have the general relation

$$
V A_{f}^{(N)} V^{*}=\frac{N+1}{4 \pi} \int_{S^{2}} f(\Omega) V|\Omega\rangle\left\langle\left.\Omega\right|_{N} V^{*} \mathrm{~d} \Omega .\right.
$$

Specializing to $V=\left.\underbrace{U \otimes \cdots \otimes U}_{N \text { times }}\right|_{\operatorname{Sym}^{N}\left(\mathbb{C}^{2}\right)}$, we obtain (see Lemma 2.6)

$$
V|\Omega\rangle=e^{i \alpha_{U, \Omega}}\left|R_{U} \Omega\right\rangle
$$

where the phase is irrelevant as it disappears in view of later computations; hence,

$$
\begin{aligned}
V A_{f}^{(N)} V^{*} & =\frac{N+1}{4 \pi} \int_{S^{2}} f(\Omega)\left|R_{U} \Omega\right\rangle\left\langle\left. R_{U} \Omega\right|_{N} \mathrm{~d} \Omega\right. \\
& =\frac{N+1}{4 \pi} \int_{S^{2}} f\left(R_{U}^{-1} \Omega\right)\left|R_{U} R_{U}^{-1} \Omega\right\rangle\left\langle\left. R_{U} R_{U}^{-1} \Omega\right|_{N} d R_{U}^{-1} \Omega,\right.
\end{aligned}
$$

namely

$$
\begin{aligned}
& \left.\left.\underbrace{U \otimes \cdots \otimes U}_{N \text { times }}\right|_{\operatorname{Sym}^{N}\left(\mathbb{C}^{2}\right)} A \underbrace{U^{*} \otimes \cdots \otimes U^{*}}_{N \text { times }}\right|_{\operatorname{Sym}^{N}\left(\mathbb{C}^{2}\right)} \\
& =\frac{N+1}{4 \pi} \int_{S^{2}} f\left(R_{U}^{-1} \Omega\right)|\Omega\rangle\left\langle\left.\Omega\right|_{N} \mathrm{~d} \Omega\right.
\end{aligned}
$$


where we took advantage of $\mathrm{d} \Omega=d R^{-1} \Omega$ if $R \in S O$ (3). To conclude, if $A=$ $Q_{1 / N}\left(p_{m}^{(j)}\right)$, identity (2.14) yields

$$
\int_{S^{2}} \sum_{m^{\prime}} D_{m m^{\prime}}^{(j)}\left(R^{-1}\right) q_{m^{\prime}}^{(j)}(\Omega)|\Omega\rangle\left\langle\left.\Omega\right|_{N} \mathrm{~d} \Omega=\int_{S^{2}} q_{m}^{(j)}\left(R_{U}^{-1} \Omega\right) \mid \Omega\right\rangle\left\langle\left.\Omega\right|_{N} \mathrm{~d} \Omega .\right.
$$

Since the map (2.2) is bijective on $P_{N}\left(S^{2}\right)$, it must be

$$
q_{m}^{(j)}\left(R^{-1} \Omega\right)=\sum_{m^{\prime}} D_{m m^{\prime}}^{(j)}\left(R^{-1}\right) q_{m^{\prime}}^{(j)}(\Omega), \quad \forall \Omega \in S^{2}, \forall R \in S O(3)
$$

Linearity and bijectivity of the map (2.5) also imply that, varying $m=-j,-j+$ $1, \ldots, j-1, j$ the functions $q_{m}^{(j)}$ form a basis of a $2 j+1$-dimensional subspace of $P_{N}\left(S^{2}\right)$. We can expand each of these functions over the basis of functions $p_{m}^{(j)}$ of $P_{N}\left(S^{2}\right)$ :

$$
q_{m^{\prime}}^{\left(j^{\prime}\right)}=\sum_{j=0}^{N} \sum_{m=-j}^{j} C_{m^{\prime} m}^{\left(j^{\prime}, j\right)} p_{m}^{(j)}
$$

where both sides are now and henceforth evaluated on $S^{2}$. Here, (2.7) and (2.17) together imply

$$
\sum_{j, m, \kappa} D_{m^{\prime} \kappa}^{(j)}(R) C_{\kappa m}^{\left(j^{\prime}, j\right)} p_{m}^{(j)}=\sum_{j, m, \ell} C_{m^{\prime} m}^{\left(j^{\prime}, j\right)} D_{m \ell}^{(j)}(R) p_{\ell}^{(j)},
$$

that is,

$$
\sum_{j, \ell, \kappa} D_{m^{\prime} \kappa}^{(j)}(R) C_{\kappa \ell}^{\left(j^{\prime}, j\right)} p_{\ell}^{(j)}=\sum_{j, m, \ell} C_{m^{\prime} m}^{\left(j^{\prime}, j\right)} D_{m \ell}^{(j)}(R) p_{\ell}^{(j)}
$$

Since the set of the (restrictions of to the sphere of the) $p^{(j)}$ is a basis,

$$
\sum_{m} D_{m^{\prime} m}^{(j)}(R) C_{m \ell}^{\left(j^{\prime}, j\right)}=\sum_{m} C_{m^{\prime} m}^{\left(j^{\prime}, j\right)} D_{m \ell}^{(j)}(R)
$$

Since the representation $D^{(j)}$ is irreducible, Schur's lemma implies that there are complex numbers $C^{\left(j^{\prime}, j\right)}$ such that

$$
C_{m \ell}^{\left(j^{\prime}, j\right)}=C^{\left(j^{\prime}, j\right)} \delta_{m \ell} .
$$

In summary, (2.19) reduces to

$$
q_{m}^{\left(j^{\prime}\right)}=\left.\sum_{j=0}^{M} C^{\left(j^{\prime}, j\right)} p_{m}^{(j)}\right|_{S^{2}}
$$


However, since the elements in the left-hand side are $2 j^{\prime}+1$, whereas for every $j$ in the right-hand side we have $2 j+1$ elements and the spaces of these representations transform separately, the only possibility is that $C^{\left(j^{\prime}, j\right)}=0$ if $j \neq j^{\prime}$. In other words,

$$
q_{m}^{(j, N)}=\left.C_{N}^{(j)} p_{m}^{(j)}\right|_{S^{2}} \quad \text { for every given } j=0,1,2, \ldots, M
$$

where

(i) we have terminated $j$ to $M<N$ because the initial polynomial $p_{m}^{(j)}$ has been chosen in $P_{M}^{(j)}\left(S^{2}\right)$;

(ii) we have restored the presence of $N$, since $C_{N}^{(j)}$ may depend on $N$.

Let us examine what happens to $C_{N}^{(j)}$ at large $N$. First observe that (2.20) immediately implies

$$
\left.Q_{1 / N}\left(p_{m}^{(j)}\right)\right|_{\operatorname{Sym}^{N}\left(\mathbb{C}^{2}\right)}=C_{N}^{(j)} \frac{N+1}{4 \pi} \int_{S^{2}} p_{m}^{(j)}(\Omega)|\Omega\rangle\left\langle\left.\Omega\right|_{N} \mathrm{~d} \Omega .\right.
$$

Taking the expectation value $\left\langle\Omega^{\prime}|\cdot| \Omega^{\prime}\right\rangle$, we find

$$
p_{m}^{(j)}\left(\Omega^{\prime}\right)=C_{N}^{(j)} \int_{S^{2}} p_{m}^{(j)}(\Omega) \frac{N+1}{4 \pi}\left|\left\langle\Omega^{\prime} \mid \Omega\right\rangle_{N}\right|^{2} \mathrm{~d} \Omega .
$$

In Lemma 2.5, we prove that $\lim _{N \rightarrow+\infty} C_{N}^{(j)}$ exists and is finite. Hence,

$$
p_{m}^{(j)}\left(\Omega^{\prime}\right)=\left(\lim _{N \rightarrow+\infty} C_{N}^{(j)}\right) p_{m}^{(j)}\left(\Omega^{\prime}\right)
$$

where we exploited Proposition 4.2 of [15], so that

$$
\lim _{N \rightarrow+\infty} C_{N}^{(j)}=1
$$

This reasoning implies the claim for the considered special polynomials since, for $N \rightarrow+\infty$,

$$
\begin{aligned}
& \|\left. Q_{1 / N}\left(p_{m}^{(j)}\right)\right|_{\operatorname{Sym}^{N}\left(\mathbb{C}^{2}\right)}-\left.\frac{N+1}{4 \pi} \int_{S^{2}} p_{m}^{(j)}\right|_{S^{2}}(\Omega)|\Omega\rangle\left\langle\left.\Omega\right|_{N} \mathrm{~d} \Omega \|_{N}\right. \\
& =\left|C_{N}-1\right| \|\left.\frac{N+1}{4 \pi} \int_{S^{2}} p_{m}^{(j)}\right|_{S^{2}}(\Omega)|\Omega\rangle\left\langle\left.\left.\Omega\right|_{N} \mathrm{~d} \Omega\left\|_{N} \leq\left|C_{N}-1\right|\right\| p_{m}^{(j)}\right|_{S^{2}} \|_{\infty} \rightarrow 0\right.
\end{aligned}
$$

The found result immediately extends to every polynomial of given degree $M$ which can be written as a linear combination of the $p_{m}^{(j)}$ viewed as polynomials. To pass to 
a generic polynomial in $\tilde{A}_{0}$ (say of degree $M$ ), we observe that, as a consequence of known results [21], the map

$$
\left.\tilde{A}_{0} \ni p \mapsto p\right|_{S^{2}} \in P_{M}\left(S^{2}\right)
$$

has a kernel made of all possible polynomials of the form $q(x, y, z)\left(x^{2}+y^{2}+z^{2}-1\right)$ with $q \in P_{M-2}$. Furthermore, Proposition 2.7 proves that, for every $q \in P_{M-2}$,

$$
\left\|\left.Q_{1 / N}\left(q(x, y, z)\left(x^{2}+y^{2}+z^{2}-1\right)\right)\right|_{\operatorname{Sym}^{N}\left(\mathbb{C}^{2}\right)}\right\|_{N} \rightarrow 0 \text { as } N \rightarrow+\infty \text {. }
$$

So, if $p \in \tilde{A}_{0}$ is a polynomial of degree $M$, then we can write for a finite number of coefficients $C^{(j, m)}$ and some polynomial $q \in P_{M-2}$,

$$
p=\sum_{j, m} C^{(j, m)} p_{m}^{(j)}+q(x, y, z)\left(x^{2}+y^{2}+z^{2}-1\right),
$$

where the $p_{m}^{(j)}$ and $q$ are here interpreted as elements of $P_{M}$ and $P_{M-2}$, respectively, restricted to $B^{3}$. Hence,

$$
\begin{gathered}
\left.Q_{1 / N}(p)\right|_{\operatorname{Sym}^{N}\left(\mathbb{C}^{2}\right)}=\left.\sum_{j, m} C^{(j, m)} Q_{1 / N}\left(p_{m}^{(j)}\right)\right|_{\operatorname{Sym}^{N}\left(\mathbb{C}^{2}\right)} \\
+\left.Q_{1 / N}\left(q(x, y, z)\left(x^{2}+y^{2}+z^{2}-1\right)\right)\right|_{\operatorname{Sym}^{N}\left(\mathbb{C}^{2}\right)} \cdot
\end{gathered}
$$

The former term on the right-hand side tends to $Q_{1 / N}^{\prime}\left(\left.p\right|_{S^{2}}\right)$, and the latter vanishes as $N \rightarrow+\infty$ proving the thesis.

\subsection{Subsidiary technical results}

Lemma $2.5 \lim _{N \rightarrow+\infty} C_{N}^{(j)}$ exists and is finite.

Proof Since the left-hand side of (2.21) does not depend on $N$ and the integral in the right-hand side tends to $p_{m}^{(j)}\left(\Omega^{\prime}\right)$, the only possibility that the $\operatorname{limit}_{N \rightarrow+\infty} C_{N}^{(j)}$ prevents from existing (or that makes it infinite) is $p_{m}^{(j)}\left(\Omega^{\prime}\right)=0$. This result should be true for all $\Omega^{\prime}$, since $\lim _{N \rightarrow+\infty} C_{N}$ is independent of $\Omega^{\prime}$. However, the polynomial $p_{m}^{(j)}$ (restricted to $S^{2}$ ) is not the zero function since it is an element of a basis.

Lemma $2.6 \mathrm{Eq}$. (2.15) is true.

Proof As is well known (see [15] for a summary of those properties and technical references),

$$
\Omega \cdot \sigma|\Omega\rangle_{1}=|\Omega\rangle_{1}
$$

Applying $U$ to both sides gives

$$
\Omega \cdot U \sigma U^{*} U|\Omega\rangle_{1}=U|\Omega\rangle_{1}
$$


Namely, from (2.11) we obtain

$$
\Omega \cdot\left(R_{U}^{-1} \sigma\right) U|\Omega\rangle_{1}=U|\Omega\rangle_{1},
$$

that is,

$$
\left(R_{U} \Omega\right) \cdot \sigma U r|\Omega\rangle_{1}=U|\Omega\rangle_{1} .
$$

We also know that

$$
\left(R_{U} \Omega\right) \cdot \sigma\left|R_{U} \Omega\right\rangle_{1}=\left|R_{U} \Omega\right\rangle_{1} .
$$

Since the eigenspace of $\left(R^{-1} \Omega\right) \cdot \sigma$ with eigenvalue 1 is one-dimensional, for some real $\beta_{\Omega, U}$, we must have

$$
U|\Omega\rangle_{1}=e^{i \beta_{\Omega, U}}\left|R_{U} \Omega\right\rangle_{1}
$$

Taking advantage of $|\Omega\rangle_{N}=\underbrace{|\Omega\rangle_{1} \otimes \cdots \otimes|\Omega\rangle_{1}}_{N \text { times }}$ and $V=\left.\underbrace{U \otimes \cdots \otimes U}_{N \text { times }}\right|_{\operatorname{Sym}^{N}\left(\mathbb{C}^{2}\right)}$, we immediately achieve (2.15) with $\alpha_{\Omega, U}=N \beta_{\Omega, U}$.

Proposition 2.7 Equations (2.23) is true.

Proof We use the canonical (Dicke) basis [15,18] $|n, N-n\rangle$ for $\operatorname{Sym}^{N}\left(\mathbb{C}^{2}\right)(n=$ $0, \ldots, N)$ and first show that the matrix elements with respect to this basis are zero:

$$
\left\langle n\left|Q_{1 / N}(q(x, y, z)) Q_{1 / N}\left(x^{2}+y^{2}+z^{2}-1\right)\right| k\right\rangle=0, \quad(k, n=0, \ldots, N) .
$$

Consider now a basis vector $|k, N-k\rangle$. We first expand $|k, N-k\rangle$ in the standard basis vectors $\beta_{i}\left(i=1, \ldots, 2^{N}\right)$ spanning the Hilbert space $\bigotimes^{N} \mathbb{C}^{2}$. We denote by $\mathscr{O}^{k}$ the orbit consisting of $\left(\begin{array}{l}N \\ k\end{array}\right)$-basis vectors $\beta_{i}$ with the same number of occurrence of the vectors $e_{2}$ and $e_{1}$, the two basis vectors of $\mathbb{C}^{2}$. By convention, we take $e_{1}$ such that $\sigma_{3} e_{1}=e_{1}$, and $\sigma_{3} e_{2}=-e_{2}$. It is not difficult to show that $[25,26]$

$$
|k, N-k\rangle=\frac{1}{\sqrt{\left(\begin{array}{c}
N \\
k
\end{array}\right)}} \sum_{l=1}^{\left(\begin{array}{c}
N \\
k
\end{array}\right)} \beta_{k, l}
$$

where the subindex $l$ in $\beta_{k, l}$ labels the basis vector $\beta_{k, l} \in \beta$ within the same orbit $\mathscr{O}^{k}$. Since we have $\left(\begin{array}{l}N \\ k\end{array}\right)$ such vectors per orbit, the sum in the above equation indeed is from $l=1, \ldots,\left(\begin{array}{c}N \\ k\end{array}\right)$. By definition, $Q_{1 / N}\left(x_{i}^{2}\right)=S_{2, N}\left(\sigma_{i} \otimes \sigma_{i}\right)$ for $i=1,2,3$. Using a combinatorial argument and the fact that all $|k\rangle$ are symmetric, it follows that 


$$
\begin{aligned}
& S_{2, N}\left(\sigma_{2} \otimes \sigma_{2}\right)|k\rangle=\frac{1}{\sqrt{\left(\begin{array}{c}
N \\
k
\end{array}\right)}} \sum_{l=1}^{\left(\begin{array}{c}
N \\
k
\end{array}\right)}\left(\sigma_{2} \otimes \sigma_{2} \otimes 1 \cdots \otimes 1\right) \beta_{k, l} \\
& =\frac{1}{\sqrt{\left(\begin{array}{c}
N \\
k
\end{array}\right)}}\left(-\left(\begin{array}{c}
N-2 \\
k-2
\end{array}\right) \beta_{k-2, l}+2\left(\begin{array}{c}
N-2 \\
k-1
\end{array}\right) \beta_{k, l}-\left(\begin{array}{c}
N-2 \\
k
\end{array}\right) \beta_{k+2, l} .\right.
\end{aligned}
$$

Similarly,

$$
\begin{aligned}
& S_{2, N}\left(\sigma_{1} \otimes \sigma_{1}\right)|k\rangle \\
& \quad=\frac{1}{\sqrt{\left(\begin{array}{l}
N \\
k
\end{array}\right)}}\left(\left(\begin{array}{l}
N-2 \\
k-2
\end{array}\right) \beta_{k-2, l}+2\left(\begin{array}{c}
N-2 \\
k-1
\end{array}\right) \beta_{k, l}+\left(\begin{array}{c}
N-2 \\
k
\end{array}\right) \beta_{k+2, l} ;\right.
\end{aligned}
$$

and

$$
\begin{aligned}
& S_{2, N}\left(\sigma_{3} \otimes \sigma_{3}\right)|k\rangle \\
& \quad=\frac{1}{\sqrt{\left(\begin{array}{c}
N \\
k
\end{array}\right)}}\left(\left(\begin{array}{c}
N-2 \\
k-2
\end{array}\right) \beta_{k, l}-2\left(\begin{array}{c}
N-2 \\
k-1
\end{array}\right) \beta_{k, l}+\left(\begin{array}{c}
N-2 \\
k
\end{array}\right) \beta_{k, l} .\right.
\end{aligned}
$$

In view of Definition 1.1 (property $3(b)$ ), the cross section $0 \rightarrow f$ and $1 / N \rightarrow$ $Q_{1 / N}(f)$ defines a continuous section of the bundle implying that the following condition (see also the remark below Definition 1.1) is automatically satisfied:

$$
\lim _{N \rightarrow \infty}\left\|Q_{1 / N}(f) Q_{1 / N}(f)-Q_{1 / N}(f g)\right\|_{N}=0
$$

We apply this with $f=q(x, y, z)$ and $g(x, y, z)=x^{2}+y^{2}+z^{2}-1$. We first show that

$$
\left.\langle n| Q_{1 / N}(q(x, y, z)) Q_{1 / N}\left(x^{2}+y^{2}+z^{2}-1\right)\right)|k\rangle=0,
$$

for all basis vectors $|n\rangle$ and $|k\rangle$ in $\operatorname{Sym}^{N}\left(\mathbb{C}^{2}\right)$. Indeed, using the above identities one finds

$$
\begin{aligned}
& \left\langle n\left|Q_{1 / N}(q(x, y, z)) Q_{1 / N}\left(x^{2}+y^{2}+z^{2}-1\right)\right| k\right\rangle \\
& =\frac{1}{\sqrt{\left(\begin{array}{c}
N \\
n
\end{array}\right)}} \frac{1}{\sqrt{\left(\begin{array}{c}
N \\
k
\end{array}\right)}} \sum_{l=1}^{\left(\begin{array}{c}
N \\
n
\end{array}\right)} \sum_{r=1}^{\left.\begin{array}{c}
N \\
k
\end{array}\right)}\left\langle\beta_{n, l}, Q_{N}(q(x, y, z))\left(S_{2, N}\left(\sigma_{1} \otimes \sigma_{1}\right)+S_{2, N}\left(\sigma_{2} \otimes \sigma_{2}\right)+S_{2, N}\left(\sigma_{3} \otimes \sigma_{3}\right)\right) \beta_{k, r}\right\rangle \\
& -\frac{1}{\sqrt{\left(\begin{array}{c}
N \\
n
\end{array}\right)}} \frac{1}{\sqrt{\left(\begin{array}{c}
N \\
k
\end{array}\right)}} \sum_{l=1}^{\left(\begin{array}{c}
N \\
n
\end{array}\right)} \sum_{r=1}^{\left(\begin{array}{c}
N \\
k
\end{array}\right)}\left\langle\beta_{n, l}, Q_{1 / N}(q(x, y, z)) \beta_{k, r}\right\rangle \\
& =\frac{1}{\sqrt{\left(\begin{array}{c}
N \\
n
\end{array}\right)}} \frac{1}{\sqrt{\left(\begin{array}{c}
N \\
k
\end{array}\right)}} \sum_{l=1}^{\left(\begin{array}{l}
N \\
n
\end{array}\right)}\left\langle\beta_{n, l}, Q_{N}(q(x, y, z))\left(\left(\begin{array}{c}
N-2 \\
k-2
\end{array}\right)+\left(\begin{array}{c}
N-2 \\
k
\end{array}\right)+2\left(\begin{array}{c}
N-2 \\
k-1
\end{array}\right)-\left(\begin{array}{l}
N \\
k
\end{array}\right)\right) \beta_{k, r}\right\rangle
\end{aligned}
$$




$$
\frac{1}{\sqrt{\left(\begin{array}{c}
N \\
n
\end{array}\right)}} \frac{1}{\sqrt{\left(\begin{array}{c}
N \\
k
\end{array}\right)}} \sum_{l=1}^{\left(\begin{array}{c}
N \\
n
\end{array}\right)}\left\langle\beta_{n, l}, Q_{N}(q(x, y, z))\left(\left(\begin{array}{l}
N \\
k
\end{array}\right)-\left(\begin{array}{l}
N \\
k
\end{array}\right)\right) \beta_{k, r}\right\rangle=0 .
$$

Since this holds for all basis vectors and $\operatorname{Sym}^{N}\left(\mathbb{C}^{2}\right)$ is invariant under $Q_{1 / N}(q(x, y, z))$ and $Q_{1 / N}\left(x^{2}+y^{2}+z^{2}-1\right)$, we conclude

$$
\left.\left(Q_{1 / N}(q(x, y, z)) Q_{1 / N}\left(x^{2}+y^{2}+z^{2}-1\right)\right)\right|_{\operatorname{Sym}^{N}\left(\mathbb{C}^{2}\right)}=0 .
$$

Therefore, for any symmetric unit vector $\phi \in \operatorname{Sym}^{N}\left(\mathbb{C}^{2}\right)$ we compute

$$
\begin{aligned}
& \left\|Q_{1 / N}\left(q(x, y, z)\left(x^{2}+y^{2}+z^{2}-1\right)\right) \phi\right\|_{N} \\
& \quad=\left\|\left(Q_{1 / N}\left(q(x, y, z)\left(x^{2}+y^{2}+z^{2}-1\right)\right)-Q_{1 / N}(q(x, y, z)) Q_{1 / N}\left(x^{2}+y^{2}+z^{2}-1\right)\right) \phi\right\|_{N} \\
& \leq\left\|Q_{1 / N}\left(q(x, y, z)\left(x^{2}+y^{2}+z^{2}-1\right)\right)-Q_{1 / N}(q(x, y, z)) Q_{1 / N}\left(x^{2}+y^{2}+z^{2}-1\right)\right\|_{N} .
\end{aligned}
$$

As a consequence of (2.26), for every $\epsilon>0$ there is $N_{\epsilon}$ such that

$$
\left\|Q_{1 / N}\left(q(x, y, z)\left(x^{2}+y^{2}+z^{2}-1\right)\right) \phi\right\|_{N}<\epsilon \text { if } N>N_{\epsilon}
$$

the crucial observation is that due to (2.26) the number $N_{\epsilon}$ does not depend on the unit vector $\phi \in \operatorname{Sym}^{N}\left(\mathbb{C}^{2}\right)$. Therefore, the above bound is uniform, and

$$
\begin{aligned}
& \left\|\left.Q_{1 / N}\left(q(x, y, z)\left(x^{2}+y^{2}+z^{2}-1\right)\right)\right|_{\operatorname{Sym}^{N}\left(\mathbb{C}^{2}\right)}\right\|_{N} \\
& =\sup _{\|\phi\|=1, \phi \in \operatorname{Sym}^{N}\left(\mathbb{C}^{2}\right)}\left\|Q_{1 / N}\left(q(x, y, z)\left(x^{2}+y^{2}+z^{2}-1\right)\right) \phi\right\|_{N} \leq \epsilon \quad \text { if } N>N_{\epsilon},
\end{aligned}
$$

which means

$$
\lim _{N \rightarrow \infty} \|\left. Q_{1 / N}\left(q(x, y, z)\left(x^{2}+y^{2}+z^{2}-1\right)\right)\right|_{\operatorname{Sym}^{N}\left(\mathbb{C}^{2}\right)}||_{N}=0 .
$$

This closes the proof of the proposition.

\section{Application to the quantum Curie-Weiss model}

We apply the previous theorem to the (quantum) Curie-Weiss model ${ }^{12}$, which is an exemplary quantum mean-field spin model. We recall that the quantum Curie Weiss

\footnotetext{
12 This model exists in both a classical and a quantum version and is a mean-field approximation to the Ising model. See, e.g., [9] for a mathematically rigorous treatment of the classical version, and $[6,11]$ for the quantum version. For our approach, the papers $[4,8,20]$ played an important role. See also [1] for a very detailed discussion of the quantum Curie-Weiss model.
} 
defined on a lattice with $N$ sites is

$$
\begin{aligned}
& h_{1 / N}^{C W}: \underbrace{\mathbb{C}^{2} \otimes \cdots \otimes \mathbb{C}^{2}}_{N \text { times }} \rightarrow \underbrace{\mathbb{C}^{2} \otimes \cdots \otimes \mathbb{C}^{2}}_{N \text { times }} \\
& h_{1 / N}^{C W}=\frac{1}{N}\left(-\frac{J}{2 N} \sum_{i, j=1}^{N} \sigma_{3}(i) \sigma_{3}(j)-B \sum_{j=1}^{N} \sigma_{1}(j)\right) .
\end{aligned}
$$

Here, $\sigma_{k}(j)$ stands for $I_{2} \otimes \cdots \otimes \sigma_{k} \otimes \cdots \otimes I_{2}$, where $\sigma_{k}$ occupies the $j$ th slot, and $J, B \in \mathbb{R}$ are given constants defining the strength of the spin-spin coupling and the (transverse) external magnetic field, respectively. Note that

$$
h_{1 / N}^{C W} \in \operatorname{Sym}\left(M_{2}(\mathbb{C})^{\otimes N}\right),
$$

where $\operatorname{Sym}\left(M_{2}(\mathbb{C})^{\otimes N}\right)$ is the range of the symmetrizer. Our interest will lie in the limit $N \rightarrow \infty$. As such, we rewrite $h_{1 / N}^{C W}$ as

$$
\begin{aligned}
h_{1 / N}^{C W} & =-\frac{J}{2 N(N-1)} \sum_{i \neq j, i, j=1}^{N} \sigma_{3}(i) \sigma_{3}(j)-\frac{B}{N} \sum_{j=1}^{N} \sigma_{1}(j)+O(1 / N) . \\
& =Q_{1 / N}\left(h_{0}^{C W}\right)+O(1 / N),
\end{aligned}
$$

where $O(1 / N)$ is meant in norm (i.e., the operator norm on each space $\left.M_{2}\left(\mathbb{C}^{2}\right)^{\otimes N}\right)$, and the classical Curie-Weiss Hamiltonian is

$$
\begin{aligned}
h_{0}^{C W}: B^{3} & \mapsto \mathbb{R} \\
h_{0}^{C W}(x, y, z) & =-\left(\frac{J}{2} z^{2}+B x\right), \quad \mathbf{x}=(x, y, z) \in B^{3},
\end{aligned}
$$

where $B^{3}=\left\{\mathbf{x} \in \mathbb{R}^{3} \mid\|\mathbf{x}\| \leq 1\right\}$ is the closed unit ball in $\mathbb{R}^{3}$.

Using these observations, we now show that the quantum Curie-Weiss Hamiltonian restricted to the symmetric space is asymptotically norm-equivalent also to the other quantization map $Q_{1 / N}^{\prime}$ applied to $\left.h_{0}^{C W}\right|_{S^{2}}$.

Theorem 3.1 One has

$$
\|\left. h_{1 / N}^{C W}\right|_{S y m^{N}\left(\mathbb{C}^{2}\right)}-\left.Q_{1 / N}^{\prime}\left(\left.h_{0}^{C W}\right|_{S^{2}}\right)\right|_{N} \rightarrow 0 \text { for } N \rightarrow \infty .
$$

Proof Using (3.4) and Theorem (2.3),

$$
\begin{aligned}
& \left\|\left.h_{1 / N}^{C W}\right|_{\operatorname{Sym}^{N}\left(\mathbb{C}^{2}\right)}-Q_{1 / N}^{\prime}\left(\left.h_{0}^{C W}\right|_{S^{2}}\right)\right\|_{N} \\
& \quad \leq\left\|\left.Q_{1 / N}\left(h_{0}^{C W}\right)\right|_{\operatorname{Sym}^{N}\left(\mathbb{C}^{2}\right)}+\left.O\left(\frac{1}{N}\right)\right|_{\operatorname{Sym}^{N}\left(\mathbb{C}^{2}\right)}-Q_{1 / N}^{\prime}\left(\left.h_{0}^{C W}\right|_{S^{2}}\right)\right\|_{N}
\end{aligned}
$$




$$
\leq\left\|\left.Q_{1 / N}\left(h_{0}^{C W}\right)\right|_{S_{y m}^{N}\left(\mathbb{C}^{2}\right)}-Q_{1 / N}^{\prime}\left(\left.h_{0}^{C W}\right|_{S^{2}}\right)\right\|_{N} \rightarrow 0 \quad(\text { as } N \rightarrow \infty)
$$

This, in particular, establishes a link between the (compressed) quantum CurieWeiss spin Hamiltonian and its classical counterpart on the sphere.

Acknowledgements The authors are grateful to Riccardo Ghiloni for useful technical discussions and to Klaas Landsman for its comments and suggestions. The authors thank an anonymous reviewer for very useful technical suggestions. Christiaan van de Ven is Marie Skłodowska-Curie fellow of the Istituto Nazionale di Alta Matematica and is funded by the INdAM Doctoral Programme in Mathematics and/or Applications co-funded by Marie Skłodowska-Curie Actions, INdAM-DP-COFUND-2015, Grant Number 713485.

Funding Open access funding provided by Università degli Studi di Trento within the CRUI-CARE Agreement.

Open Access This article is licensed under a Creative Commons Attribution 4.0 International License, which permits use, sharing, adaptation, distribution and reproduction in any medium or format, as long as you give appropriate credit to the original author(s) and the source, provide a link to the Creative Commons licence, and indicate if changes were made. The images or other third party material in this article are included in the article's Creative Commons licence, unless indicated otherwise in a credit line to the material. If material is not included in the article's Creative Commons licence and your intended use is not permitted by statutory regulation or exceeds the permitted use, you will need to obtain permission directly from the copyright holder. To view a copy of this licence, visit http://creativecommons.org/licenses/by/4.0/.

\section{Appendix A: Continuous bundle of $C^{*}$-algebras}

For any unital $C^{*}$-algebra $B$, the following fibers may be turned into a continuous bundle of $C^{*}$-algebras over the base space $I=\{0\} \cup 1 / \mathbb{N} \subset[0,1]$ (with relative topology, so that $(1 / N) \rightarrow 0$ as $N \rightarrow \infty)$ :

$$
\begin{aligned}
A_{0} & =C(S(B)) ; \\
A_{1 / N} & =B^{\otimes N} .
\end{aligned}
$$

Here, $S(B)$ is the (algebraic) state space of $B$ equipped with the weak ${ }^{*}$-topology (in which it is a compact convex set, e.g., the three-ball $\left.S\left(M_{2}(\mathbb{C})\right) \cong B^{3} \subset \mathbb{R}^{3}\right)$, and $B^{\otimes N}$ is the $N$ th tensor power of $B$ also denoted by $B^{N}$ in what follows). ${ }^{13}$ As in the case of vector bundles, the continuity structure of a bundle of $C^{*}$-algebras may be defined (indirectly) by specifying what the continuous cross sections are. To do so for (A.1)-(A.2), we need the symmetrization operator $S_{N}: B^{N} \rightarrow B^{N}$, defined as the unique linear continuous extension of the following map on elementary tensors:

$$
S_{N}\left(a_{1} \otimes \cdots \otimes a_{N}\right):=\frac{1}{N !} \sum_{\sigma \in \mathcal{P}(N)} a_{\sigma(1)} \otimes \cdots \otimes a_{\sigma(N)} .
$$

\footnotetext{
13 Although this is irrelevant for our main application $B=M_{k}(\mathbb{C})$, for general $C^{*}$-algebras $B$, one should equip $B^{N}$ with the minimal $C^{*}$-norm \|\|$_{N}[14,24]$.
} 
Furthermore, for $N \geq M$ we need to generalize the definition of $S_{N}$ to give a bounded operator $S_{M, N}: B^{M} \rightarrow B^{N}$, defined by linear and continuous extension of

$$
S_{M, N}(b):=S_{N}(b \otimes \underbrace{I \otimes \cdots \otimes I}_{N-M \text { times }}), \quad b \in B^{\otimes M} .
$$

We write cross sections $a$ of (A.1)-(A.2) as sequences $\left(a_{0}, a_{1 / N}\right)_{N \in \mathbb{N}}$, where $a(0)=$ $a_{0}$, etc. Following [20], the part of the cross section $\left(a_{1 / N}\right)_{N \in \mathbb{N}}$ away from zero (i.e., with $a_{0}$ omitted) is called symmetric if there exist $M \in \mathbb{N}$ and $a_{1 / M} \in B^{\otimes M}$ such that

$$
a_{1 / N}=S_{M, N}\left(a_{1 / M}\right) \text { for all } N \geq M,
$$

and quasi-symmetric if $a_{1 / N}=S_{N}\left(a_{1 / N}\right)$ if $N \in \mathbb{N}$, and for every $\epsilon>0$, there is a symmetric sequence $\left(b_{1 / N}\right)_{N \in \mathbb{N}}$ as well as $M \in \mathbb{N}$ (both depending on $\epsilon$ ) such that

$$
\left\|a_{1 / N}-b_{1 / N}\right\|<\epsilon \text { for all } N>M \text {. }
$$

The continuous cross sections of the bundle (A.1) - (A.2), then, are the sequences $\left(a_{0}, a_{1 / N}\right)_{N \in \mathbb{N}}$ for which the part $\left(a_{1 / N}\right)_{N \in \mathbb{N}}$ away from zero is quasi-symmetric and

$$
a_{0}(\omega)=\lim _{N \rightarrow \infty} \omega^{N}\left(a_{1 / N}\right)
$$

where $\omega \in S(B)$, and $\omega^{N}=\underbrace{\omega \otimes \cdots \otimes \omega}_{N \text { times }} \in S\left(B^{\otimes N}\right)$, is the unique (norm) continuous linear extension of the following map that is defined on elementary tensors:

$$
\omega^{N}\left(b_{1} \otimes \cdots \otimes b_{N}\right)=\omega\left(b_{1}\right) \cdots \omega\left(b_{N}\right) .
$$

The limit in (A.7) exists provided $\left(a_{1 / N}\right)_{N \in \mathbb{N}}$ is quasi-symmetric (as we assume), and by [14, Theorem 8.4 ], this choice of continuous cross sections uniquely defines (or identifies) a continuous bundle of $C^{*}$-algebras over $I$ in (1.9) with fibers (A.1)-(A.2).

\section{Appendix B: Coherent spin states}

If $|\uparrow\rangle,|\downarrow\rangle$ are the eigenvectors of $\sigma_{3}$ in $\mathbb{C}^{2}$, so that $\sigma_{3}|\uparrow\rangle=|\uparrow\rangle$ and $\sigma_{3}|\downarrow\rangle=-|\downarrow\rangle$, and where $\Omega \in S^{2}$, with polar angles $\theta_{\Omega} \in(0, \pi), \phi_{\Omega} \in(-\pi, \pi)$, we then define the unit vector

$$
|\Omega\rangle_{1}=\cos \frac{\theta_{\Omega}}{2}|\uparrow\rangle+e^{i \phi_{\Omega}} \sin \frac{\theta_{\Omega}}{2}|\downarrow\rangle .
$$


If $N \in \mathbb{N}$, the associated $N$-coherent spin state $|\Omega\rangle_{N} \in \operatorname{Sym}^{N}\left(\mathbb{C}^{2}\right)$, equipped with the usual scalar product $\langle\cdot, \cdot\rangle_{N}$ inherited from $\left(\mathbb{C}^{2}\right)^{N}$, is defined as follows [18]:

$$
|\Omega\rangle_{N}=\underbrace{|\Omega\rangle_{1} \otimes \cdots \otimes|\Omega\rangle_{1}}_{N \text { times }} .
$$

An important property relevant for our computations was established in [15]

$$
f\left(\Omega^{\prime}\right)=\lim _{N \rightarrow \infty} \frac{N+1}{4 \pi} \int_{S^{2}} \mathrm{~d} \Omega f\left(\Omega^{\prime}\right)\left|\left\langle\Omega, \Omega^{\prime}\right\rangle_{N}\right|^{2}, \quad\left(f \in C\left(S^{2}\right)\right) .
$$

\section{References}

1. Allahverdyana, A.E., Balian, R., Nieuwenhuizen, ThM: Understanding quantum measurement from the solution of dynamical models. Phys. Rep. 525, 1-166 (2013)

2. Bengtsson, I., Zyczkowski, K.: Geometry of Quantum States: An Introduction to Quantum Entanglement. Cambridge University Press, Cambridge (2006)

3. Berezin, F.A.: General concept of quantization. Commun. Math. Phys. 40, 153-174 (1975)

4. Bona, P.: The dynamics of a class of mean-field theories. J. Math. Phys. 29, 2223-2235 (1988)

5. Bordemann, M., Meinrenken, E., Schlichenmaier, M.: Toeplitz quantization of Kähler manifolds and $g l(N), N \rightarrow \infty$ limits. Commun. Math. Phys. 165, 281-296 (1994)

6. Chayes, L., Crawford, N., Ioffe, D., Levit, A.: The phase diagram of the quantum Curie-Weiss model. J. Stat. Phys. 133, 131-149 (2008)

7. Dixmier, J.: C*-Algebras. North-Holland, Amsterdam (1977)

8. Duffield, N.G., Werner, R.F.: Local dynamics of mean-field quantum systems. Helv. Phys. Acta 65, 1016-1054 (1992)

9. Friedli, S., Velenik, Y.: Statistical Mechanics of Lattice Systems: A Concrete Mathematical Introduction. Cambridge University Press, Cambridge (2017)

10. Helffer, B.: Semi-classical Analysis for the Schrödinger Operator and Applications. Springer, Heidelberg (1988)

11. Ioffe, D., Levit, A.: Ground states for mean field models with a transverse component. J. Stat. Phys. 151, 1140-1161 (2013)

12. Kutzner, J.: Eine Phasenraumdarstellung ftir Spinsysteme. Z. Physik 259, 177-188 (1973)

13. Landsman, N.P.: Mathematical Topics Between Classical and Quantum Theory. Springer, New York (1998)

14. Landsman, K.: Foundations of Quantum Theory: From Classical Concepts to Operator Algebras Springer, Berlin (2017). Open Access at http://www.springer.com/gp/book/9783319517766

15. Landsman, K., Moretti, V., van de Ven, C.J.F.: A strict deformation quantization map on the state space of $M_{k}(\mathbb{C})$ and the classical limit of the Curie-Weiss model. Rev. Math. Phys. 32, 2050031 (2020). https://doi.org/10.1142/S0129055X20500312. arXiv:1909.10947

16. Lieb, E.H.: The classical limit of quantum spin systems. Commun. Math. Phys. 62, 327-340 (1973)

17. Moretti, V.: Spectral Theory and Quantum Mechanics, 2nd edn. Springer, New York (2018)

18. Perelomov, A.M.: Coherent states for arbitrary Lie groups. Commun. Math. Phys. 26, 222-236 (1972)

19. Pflaum, M.J.: Analytic and Geometric Study of Stratified Spaces. Springer, Berlin (2001)

20. Raggio, G.A., Werner, R.F.: Quantum statistical mechanics of general mean field systems. Helv. Phys. Acta 62, 980-1003 (1989)

21. Reimer, M.: Multivariate Polynomial Approximation. Springer, Berlin (2003)

22. Rieffel, M.A.: Deformation quantization of Heisenberg manifolds. Commun. Math. Phys. 121, 531562 (1989)

23. Rieffel, M.A.: Quantization and $C^{*}$-algebras. Contemp. Math. 167, 67-97 (1994)

24. Takesaki, M.: Theory of Operator Algebras I, 2nd edn. Springer, New York (2002)

25. van de Ven, C.J.F.: Properties of Quantum Spin Systems and their Classical Limit. M.Sc. Thesis, Radboud University (2018). https://www.math.ru.nl/ landsman/Chris2018.pdf 
26. van de Ven, C.J.F., Groenenboom, G.C., Reuvers, R., Landsman, N.P.: Quantum spin systems versus Schrödinger operators: A case study in spontaneous symmetry breaking, arXiv:1811.12109 (v3). SciPost

27. Weyl, H.: Gruppentheorie und Quantenmechanik. Hirzel, Zürich (1928)

Publisher's Note Springer Nature remains neutral with regard to jurisdictional claims in published maps and institutional affiliations. 